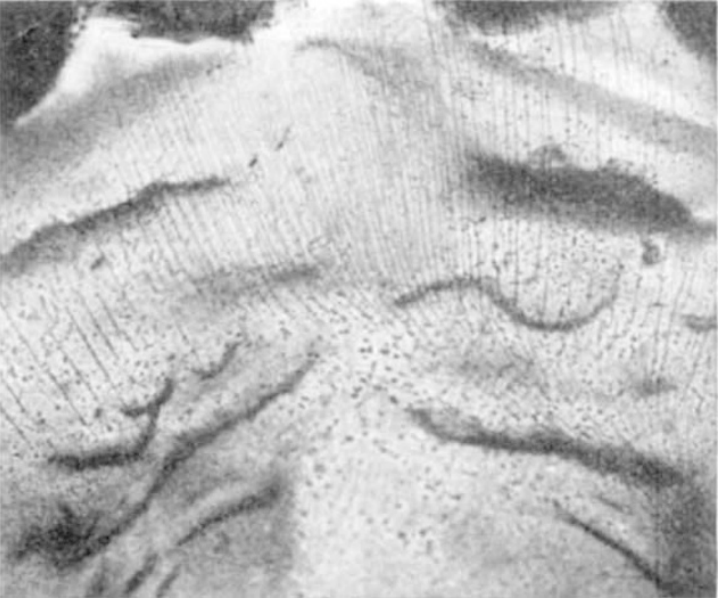

Fig. 1

region immediately behind the central incisors, shows an area of high stress, as indicated by a crowding of the crack lines. If a clear denture of polymethyl methacrylate is used, the cracks may be illuminated for photographic purposes, either by transmitted light (Fig. 1) or reflected light. Small areas of the main pattern may be photographed using reflected light, whoreas larger areas may be photographed using transmitted light with slight loss of definition.

As yet, only qualitative analysis of the crack pattern on denture bases has been attempted until more information is available about the behaviour of the lacquer on plastic surfaces. Used qualitatively, however, the lacquer provides a quick method of indicating; $(a)$ the points of high stress concentration; and $(b)$ the direction of the principal stresses, since a crack runs perpendicular to the direction of the principal stress, which is a maximum and tensile. This information is sufficient to allow strain gauges to be placed on the surface at the most advantageous points. It also allows for the orientation of the gauges, one being placed in the direction of the major principal stress, the other at right-angles in the direction of the minor principal stress. From a knowledge of the strains in these two directions, together with the elastic constants of the material, the major and minor principal stresses may be computed.

Acknowledgments are due to Dr. J. H. Lamble, senior lecturer, Department of Mechanical Engineering, University of Manchester, for his assistance. This work is being carried out under the supervision of Prof. E. Matthews.

Turner Dental School,

University of Manchester. Jan. 26.

\section{The Amorphous Alloy}

IN 1920 Rosenhain, Haughton and Bingham ${ }^{1}$ described a zinc/aluminium/copper alloy $(89 / 7 / 4)$ the mechanical properties of which resembled those of an amorphous material, in that it was ductile at room temperature when deformed slowly but brittle when deformed quickly. A simple demonstration of this was that a piece of sheet made from the metal would bend double if bent slowly but snapped if bent quickly, just like an amorphous material such as pitch. It appears that no satisfactory explanation of this behaviour has been put forward that conforms with modern ideas.

Impact tests have been made on specimens of this alloy supplied by Mr. A. E. L. Tate, who demonstrated that, in sheet form, it behaved as described above. The impact tests were made in a Charpy machine on notched specimens (for convenience the notches were of the Izod type) over a range of temperatures from about $-200^{\circ} \mathrm{C}$. to $+300^{\circ} \mathrm{C}$.; for the tests above room temperature, specimens were immersed for $15 \mathrm{sec}$. in oil maintained at the desired temperature and then tested in about $4 \mathrm{sec}$. The results are shown in Fig. 1 .

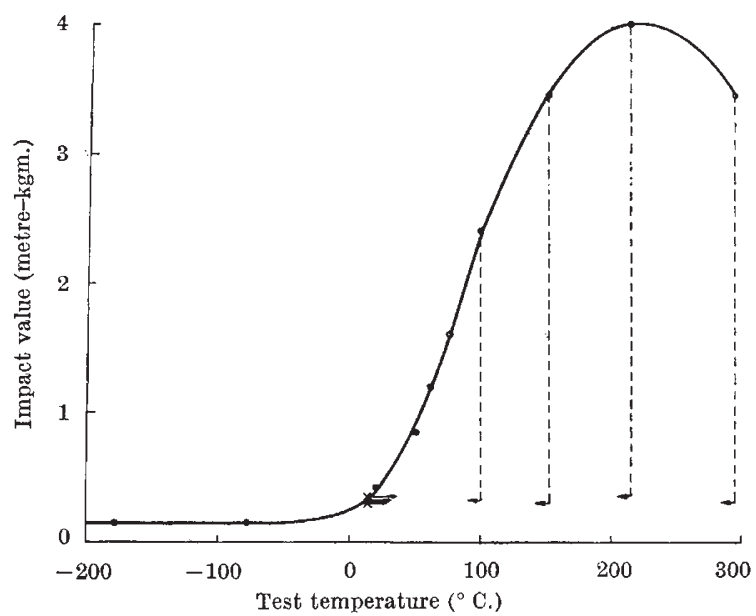

Fig. 1

The significant feature is the tough-brittle transition that takes place in the range $+200^{\circ}$ to $0^{\circ} \mathrm{C}$. To check that this was not due to a structural change brought about by warming, duplicate specimens were quenched into water at $13^{\circ} \mathrm{C}$. from the different temperatures and quickly tested; the values so obtained are shown by crosses and clearly lie on the main curve, signifying that no structural change had taken place that affected the impact value. The alloy therefore shows a genuine tough-brittle transition.

A property of the tough-brittle transition phenomenon is that the temperature range of the transition depends on the speed of testing; it falls as the speed is decreased. The fact that this alloy shows a tough-brittle transition in the neighbourhood of room temperature then explains the behaviour described in the first paragraph : in a fast test such as rapid bending, the transition range is high and room temperature lies in the brittle region; in a slow test such as gradual bending, the transition range is lower and room temperature lies in a more ductile region.

The work described here has been carried out as part of the general research programme of the National Physical Laboratory, and this communication is published by permission of the Director of the Laboratory.

D. MCLEaN

Metallurgy Division,

National Physical Laboratory, Teddington.

April 19.

1 Rosenhain, W., Haughton, J. L., and Bingham, K. E., J. Inst. Met.., $23,26(1920)$. 